\title{
Post-developmental extracellular proteoglycan maintenance in attractin-deficient mice
}

\author{
Abdallah Azouz ${ }^{1}$ and Jonathan S. Duke-Cohan ${ }^{2,3^{*}}$ (1)
}

\begin{abstract}
Objective: Neurodegeneration and hair pigmentation alterations in mice occur consequent to aberrations at the Atrn locus coding for the transmembrane form of attractin. Earlier results pointed to a possible involvement in intracellular trafficking/export of secretory vesicles containing proteoglycan. Here we examined kidney and liver, both heavily dependent upon proteoglycan, of attractin-deficient mice to determine whether abnormalities were observed in these tissues.

Results: Histological and histochemical analysis to detect glycosylated protein identified a severe loss in attractindeficient mice of extracellular proteoglycan between kidney tubules in addition to a loss of glycosylated material within the intratubular brush border. In the liver, extracellular matrix material was significantly depleted between hepatocytes together with swollen sinuses and aberrations in the proteoglycan-dependent space of Disse. These results are consistent with a generalized defect in extracellular proteoglycan deposition in Atrn-mutant mice and support previous reports suggesting a role for attractin in the secretory vesicle pathway.
\end{abstract}

Keywords: Attractin, Extracellular matrix, Histology, Kidney, Liver, Proteoglycan

\section{Introduction}

Attractin, initially discovered as a human secreted glycoprotein circulating at high concentrations in the periphery and enabling $\mathrm{T}$ cell-monocyte clustering [1], also exists as a transmembrane form produced by alternative splicing of the ATRN gene, while the mouse only produces the transmembrane form [2, 3]. On activated $\mathrm{T}$ cells, attractin moves in electron-dense proteoglycanrich vesicles to the plasma membrane leading to transient extracellular expression [1, 4]. Mutations at the Atrn locus in the mouse result in the mahogany phenotype where, despite normal levels of the agouti protein that acts as an antagonist of $\alpha$-melanocyte stimulating

*Correspondence: Jonathan_Duke-Cohan@dfci.harvard.edu

2 Department of Medical Oncology, Dana-Farber Cancer Institute, JF517,

450 Brookline Avenue, Boston, MA 02215, USA

Full list of author information is available at the end of the article hormone $(\alpha-\mathrm{MSH})$, the agouti protein does not appear to be appropriately presented to the Melanocortin-1 receptor (Mc1R) and black/brown eumelanin synthesis persists rather than that of the lighter yellowish phaeomelanin [2]. Attractin's role in agouti presentation remains to be fully elucidated. One possibility is that the membraneanchored ectodomain may help present agouti protein by binding the positive $\mathrm{N}$-terminal leaving the $\mathrm{C}$-terminal free to interact with the Mc1R [5].

Attractin's functional range has widened following reports that mahogany $\left(A t r n^{\mathrm{mg}-3 \mathrm{~J} / \mathrm{mg}-3 \mathrm{~J}}\right)$ mice present a juvenile-onset Central Nervous System (CNS)-confined neurodegeneration characterized by hypomyelination, axonal swelling, spongiform vacuolation and microtremors [5]. This neural phenotype is found not only in other mouse mutant Atrn alleles [6,7] but also in the zitter and myelin vacuolation rats $[8,9]$, and the black tremor hamster [10], all now confirmed as Atrn mutants. The

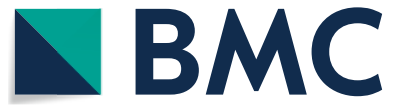

(c) The Author(s) 2020. This article is licensed under a Creative Commons Attribution 4.0 International License, which permits use, sharing, adaptation, distribution and reproduction in any medium or format, as long as you give appropriate credit to the original author(s) and the source, provide a link to the Creative Commons licence, and indicate if changes were made. The images or other third party material in this article are included in the article's Creative Commons licence, unless indicated otherwise in a credit line to the material. If material is not included in the article's Creative Commons licence and your intended use is not permitted by statutory regulation or exceeds the permitted use, you will need to obtain permission directly from the copyright holder. To view a copy of this licence, visit http://creativecommons.org/licenses/by/4.0/. The Creative Commons Public Domain Dedication waiver (http://creativecommons.org/publicdomain/zero/1.0/) applies to the data made available in this article, unless otherwise stated in a credit line to the data. 
pigmentation phenotype and neuropathology are corrected in animals transgenic for membrane attractin [5, 8]. Embryonic development appears normal in Atrnmutant mice; the neurodegeneration is manifest during juvenile maturation and may be related to a defect in maintaining the integrity of the plasma membrane with potentially severe consequences for oligodendrocytedirected myelination [4, 11]. Attractin's common biochemical role in immune cell interactions, regulation of pigmentation and neural pathology remains undefined. A function in vesicular transport of cargo, both proteoglycan and new lipid-raft rich membrane, to the plasma membrane is implicated.

\section{Main text Methods \\ Mice}

Age-matched male $\mathrm{C} 3 \mathrm{HeB} / \mathrm{FeJ}$ mice and $\mathrm{C} 3 \mathrm{HeB} / \mathrm{FeJ}-$ Atrn ${ }^{\mathrm{mg}-3 \mathrm{~J} / \mathrm{mg}-3 \mathrm{~J}}$ homozygotes were obtained from the Jackson Laboratory (Bar Harbor, ME). Animals were housed maximum 3 to a cage according to institutional guidelines in an Association for Assessment and Accreditation of Laboratory Animal Care (AAALAC)-accredited facility at the Dana-Farber Cancer Institute. Since Atrn mutations are recessive, homozygous Atrn $^{\mathrm{mg}-3 \mathrm{~J} /}$ mg-3J mice were mated with heterozygous Atrn $^{+/ \mathrm{mg}-3 \mathrm{~J}}$ mice resulting in litters where half the mice were wildtype phenotype and half the mice were recessive Atrn mutants. In any experiment, only control and mutant siblings from the same mating were compared (aged 3-3.5 months), and comparisons examined mice with no gender preference. Euthanasia was by $\mathrm{CO}_{2}$ inhalation followed by cervical dislocation with all procedures approved under Dana-Farber Cancer Institute Animal Care and Use Committee (ACUC) protocol 99-026.

\section{Histology}

Organs were excised and fixed in Bouin's fluid, formalin, or methanol depending upon the subsequent staining protocol. Tissues were then embedded in paraffin and $4 \mu \mathrm{m}$ sections were arranged on glass slides. Hematoxylin and eosin $(\mathrm{H} \& \mathrm{E})$ staining followed standard histopathology procedures. For detection of glycoprotein, rehydrated sections were placed in periodic acid $(0.5 \%$ in water) for 15 min, rinsed, placed in Schiff's reagent ( $0.5 \%$ in water; Sigma, St Louis MO), developed in running water and counterstained with hematoxylin prior to mounting. Photomicroscopic images were digitally captured using the "Magnafire" system (Olympus, Melville NY).

\section{Proteoglycan quantification}

To quantitate relative proteoglycan staining using Periodic acid-Schiff reagent (PAS) staining in sections from control and attractin-deficient mice, histology images were imported as JPG files into Image J [12]. After setting the appropriate scale (in $\mu \mathrm{m}$ ), the images were deconvoluted into red, blue and green (RGB) layers and converted into grey-scale images where the green layer gives the clearest distinction between PAS stain and background. Using the green layers, thresholds were set to exclude background signal, signal above threshold was identified and fractional representation of marked pixels in the image was recorded. For every paired analysis of tissues from control and attractin-deficient mice, identical image processing and set thresholds were used.

\section{Results}

In this report we demonstrate that attractin-deficient mahogany mice, despite apparently normal gross organ structure, have a severe juvenile-onset progressive loss of basement membrane within organs heavily dependent upon extracellular matrix (ECM) function. Our attention was drawn to overall organ structure by the consistent observation that the spleens of older Atrn ${ }^{\mathrm{mg}-3 \mathrm{~J} / \mathrm{mg}-3 \text { J }}$ mice ( $\sim 5$ months or more) are half to two-thirds the size of spleens from wild-type or heterozygous littermates (Fig. 1). Since the neuropathology in Atrn-null mice is moderate-to-severe at 2-4 months of age, we examined mice 3 to 3.5 months of age to determine if degeneration was occurring in tissues other than brain or spleen, including the kidney, liver and thymus. At this age, spleen size, cellularity and differential lymphocyte counts are comparable for wild-type and the Atrn mutants (data not presented). Kidney histology showed that the organization of individual nephrons seemed normal (Fig. 2a, c), but the interstitial matrix connecting the tubules was missing (Fig. 2b, d). Normal kidney stained well with PAS reagent (Fig. 2e, Additional file 1: Figure S1A) but not with Alcian blue (data not presented) indicating that

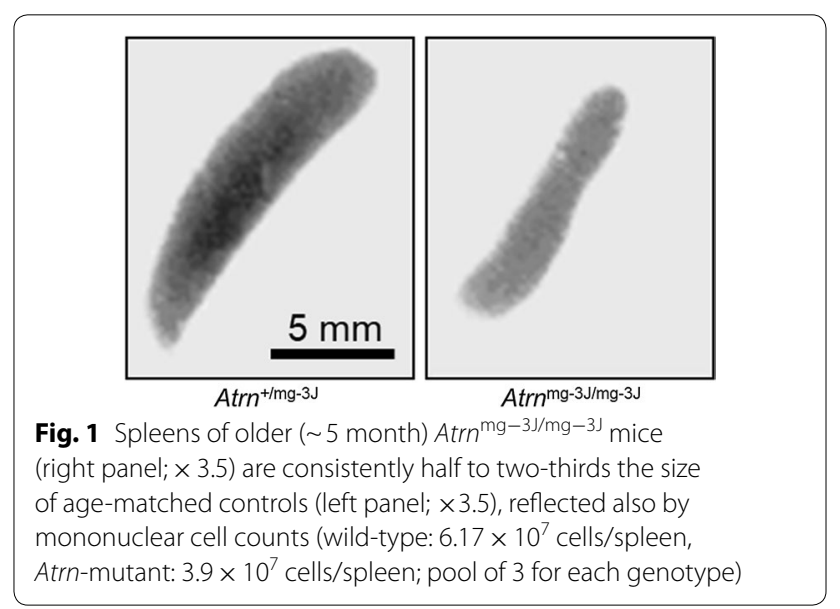




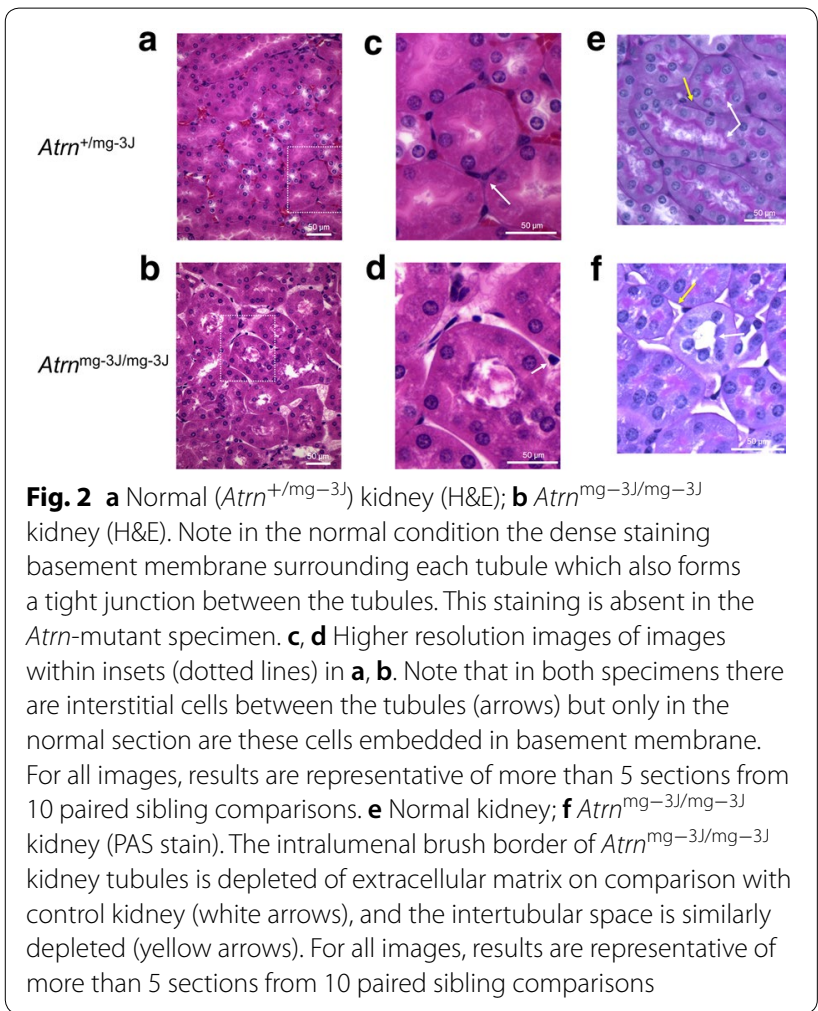

the normal interstitial matrix contains substantial levels of glycosylated protein including proteoglycan but little acid mucopolysaccharide. In contrast, kidney from Atrn mutants did not stain well with PAS indicating reduced extracellular glycosylated material (Fig. 2f, Additional file 1: Figure S1b). Quantitative analysis assessed glycosylation as $31.1 \%$ across the control kidney section and 4.4\% across the attractin-deficient kidney section (Supplemental Fig. 1c, d). Since the mutant signal coincided primarily with a strong haematoxylin nuclear signal, this is likely to be a background also for the control section. In contrast to the control, attractin-deficient kidney samples exhibited an almost complete loss of the extracellular matrix between the tubules, as well as similar loss of the proteoglycan-rich brush border within the tubules (Fig. 2e, f). There is not a generalized effect upon glycosylation within the mutant cells. Glycosylation of secreted proteins such as agouti destined for the extracellular compartment appears normal in $A t r n^{\mathrm{mg}-3 \mathrm{~J} / \mathrm{mg}-3 \mathrm{~J}}$ mice [5]. Although a role for attractin in regulating specific glycosyltransferases cannot be excluded, the observed defects are consistent with a fault in extracellular matrix/proteoglycan secretion and deposition. In support, in the liver we find that the basement membrane between hepatocytes of wild-type mice is heavily stained (Fig. 3a, c) but the ECM between the hepatocytes of Atrn ${ }^{\mathrm{mg}-3 \mathrm{~J} / \mathrm{mg}-3 \mathrm{~J}}$ mice is strongly reduced (Fig. $3 \mathrm{~b}, \mathrm{~d}$ ). Note the absence

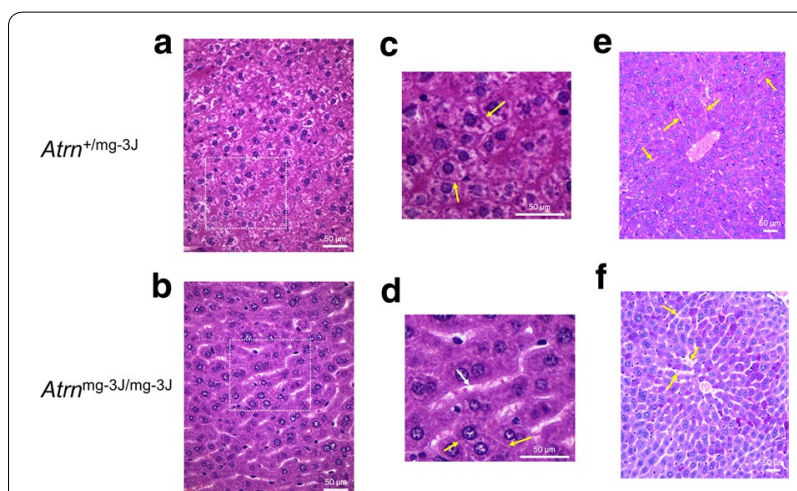

Fig. 3 a Normal liver and $\mathbf{b}$ Atrn ${ }^{\mathrm{mg}-3 \mathrm{~J} / \mathrm{mg}-3 \mathrm{~J}}$ liver (H\&E stain). The sinusoids (white space between rows of hepatocytes) are readily apparent in the mutant mice. Glycogen does not stain with H\&E leading to light areas within the control cells, an effect not seen in the mutant indicating depletion of glycogen. $\mathbf{c}, \mathbf{d}$ Higher resolution images of images within insets (dotted lines) in $\mathbf{a}, \mathbf{b}$. White arrow identifies sinusoid, yellow arrows indicate intercellular space. e Normal liver; $\mathbf{f}$ Atrn ${ }^{\mathrm{mg}-3 \mathrm{~J} / \mathrm{mg}-3 \mathrm{~J}}$ liver (PAS stain). Comparison of sinusoids (yellow arrows) clearly indicates presence of proteoglycan contributing to the perisinusoidal space of Disse in the control liver while such signal is absent in the attractin-deficient liver. Note also (at the yellow arrows) evidence for sinusoidal swelling in the Atrn ${ }^{\mathrm{mg}-3 \mathrm{~J} /}$ mg-3J liver accompanied by a more general lack of inter-hepatocyte ECM on comparison with control

of glycogen in the mutant hepatocytes, probably consequent to the higher basal metabolic rate associated with the neurodegeneration-induced tremor [7]. Using PAS staining, in contrast to the control condition (Fig. 3e, Additional file 1: Figure S2A), the liver of $A t r n^{\mathrm{mg}-3 \mathrm{~J} / \mathrm{mg}-33}$ also appears to be relatively deficient in glycosylated protein (Fig. 3f, Additional file 1: Figure S2B). Quantitative analysis assessed glycosylation as $26.7 \%$ across the control liver section and $10.0 \%$ across the attractin-deficient liver section (Additional file 1: Figure S2C, D). For the mutant liver samples, the positive signal represented predominantly a background high nuclear staining by haematoxylin. Further, the sinuses are swollen with reduced presence of the ECM-dependent space of Disse (perisinusoidal space) (Fig. 3e, f). In the thymus, where we believe attractin plays little role based on mRNA expression [1], there is no observable histological difference between control and mutant animals (data not shown).

\section{Discussion}

We have been unable to demonstrate any interaction of either natural or recombinant attractin ectodomain with any component of the ECM, and attractin does not appear to be a component of the ECM. We propose that attractin functions in proteoglycan-rich granule exocytosis and ECM maintenance in the differentiated state, a process that will replenish the plasma membrane with 
new membrane as exocytosis occurs. Given its location in electron-dense granule-rich secretory vesicles [1], attractin may have evolved a secondary function for aiding transport to the exterior of positively charged peptides including agouti and certain chemokines. The reduction or absence of ECM-proteoglycan would have profound effects upon the presentation of basic peptides and chemokines that may account in part for the immune and pigmentation-related functionality of attractin [13, 14]. The proposed role for vesicular trafficking attractin affecting ECM deposition and plasma membrane maintenance provides a unifying hypothesis for the pleiotropic effects of the null genotype and identifies avenues for further exploration. An additional consideration is that as yet unclassified human pathologies that involve neurodegeneration and concomitant renal dysfunction might be examined for abnormalities either at the ATRN locus or else its transcriptional control [15].

\section{Limitations}

Since these results describe pathology associated with a mutation in the Atrn gene, the only limitation concerns genetic penetrance. We have observed these results in the two most severe Atrn mutations $\left(A t r n^{\mathrm{mg}-3 \mathrm{~J} / \mathrm{mg}-3)}\right.$ and $A t r n^{\mathrm{mg}-6 \mathrm{~J} / \mathrm{mg}-6 \mathrm{~J}}$ ) but have not examined the Atrn ${ }^{\mathrm{mg} /}$ mg and $A t r n^{\mathrm{mg}-\mathrm{L} / \mathrm{mg}-\mathrm{L}}$ variants, strains with less severe effects upon levels of normal Atrn transcript [7].

\section{Supplementary information}

Supplementary information accompanies this paper at https://doi. org/10.1186/s13104-020-05130-1.

Additional file 1: Figure S1. Quantification of glycoprotein in wild-type and attractin-deficient kidney (PAS stain). Figure S2. Quantification of glycoprotein in wild-type and attractin-deficient liver (PAS stain).

\section{Abbreviations}

a-MSH: a-Melanocyte Stimulating Hormone; CNS: Central Nervous System; ECM: Extracellular Matrix; H\&E: Haematoxylin and Eosin; ACUC: Animal Care and Use Committee; Mc1R: Melanocortin-1 Receptor; PAS: Periodic Acid-Schiff reagent; RGB: Red/Green/Blue.

\section{Acknowledgements}

Not applicable.

\section{Authors' contributions}

AA performed the experiments and pathology analysis. JSD-C directed the study, processed data and wrote the manuscript.

\section{Funding}

Not applicable.

\section{Availability of data and materials}

Data sharing is not applicable to this article as no datasets were generated or analysed during the current study.

\section{Ethics approval and consent to participate}

Animal studies were performed under Dana-Farber Cancer Institute Animal Care and Use Committee protocol 99-026.
Consent for publication

Not applicable.

\section{Competing interests}

The authors declare that they have no competing interests.

\section{Author details}

${ }^{1}$ Department of Pathology, Regional One Health, University of Tennessee Health Science Center, Memphis, TN, USA. ${ }^{2}$ Department of Medical Oncology, Dana-Farber Cancer Institute, JF517, 450 Brookline Avenue, Boston, MA 02215, USA. ${ }^{3}$ Department of Medicine, Harvard Medical School, Boston, MA, USA.

Received: 3 April 2020 Accepted: 4 June 2020

Published online: 24 June 2020

\section{References}

1. Duke-Cohan JS, Gu J, McLaughlin DF, Xu Y, Freeman GJ, Schlossman SF. Attractin (DPPT-L), a member of the CUB family of cell adhesion and guidance proteins, is secreted by activated human T lymphocytes and modulates immune cell interactions. Proc Natl Acad Sci USA. 1998;95(19):11336-41.

2. Gunn TM, Miller KA, He L, Hyman RW, Davis RW, Azarani A, et al. The mouse mahogany locus encodes a transmembrane form of human attractin. Nature. 1999;398(6723):152-6.

3. Tang W, Gunn TM, McLaughlin DF, Barsh GS, Schlossman SF, Duke-Cohan JS. Secreted and membrane attractin result from alternative splicing of the human ATRN gene. Proc Natl Acad Sci USA. 2000;97(11):6025-30.

4. Azouz A, Gunn TM, Duke-Cohan JS. Juvenile-onset loss of lipid-raft domains in attractin-deficient mice. Exp Cell Res. 2007:313(4):761-71.

5. He L, Gunn TM, Bouley DM, Lu XY, Watson SJ, Schlossman SF, et al. A biochemical function for attractin in agouti-induced pigmentation and obesity. Nat Genet. 2001;27(1):40-7.

6. Bronson RT, Donahue LR, Samples R, Kim JH, Naggert JK. Mice with mutations in the mahogany gene Atrn have cerebral spongiform changes. J Neuropathol Exp Neurol. 2001;60(7):724-30.

7. Gunn TM, Inui T, Kitada K, Ito S, Wakamatsu K, He L, et al. Molecular and phenotypic analysis of Attractin mutant mice. Genetics. 2001;158(4):1683-95.

8. Kuramoto T, Kitada K, Inui T, Sasaki Y, Ito K, Hase T, et al. Attractin/ mahogany/zitter plays a critical role in myelination of the central nervous system. Proc Natl Acad Sci USA. 2001;98(2):559-64.

9. Kuwamura M, Maeda M, Kuramoto T, Kitada K, Kanehara T, Moriyama M et al. The myelin vacuolation ( $\mathrm{mv}$ ) rat with a null mutation in the attractin gene. Lab Invest. 2002;82(10):1279-86.

10. Kuramoto T, Nomoto T, Fujiwara A, Mizutani M, Sugimura T, Ushijima T. Insertional mutation of the Attractin gene in the black tremor hamster. Mamm Genome. 2002;13(1):36-40.

11. Tang W, Duke-Cohan JS. Human secreted attractin disrupts neurite formation in differentiating cortical neural cells in vitro. J Neuropathol Exp Neurol. 2002;61(9):767-77.

12. Schneider CA, Rasband WS, Eliceiri KW. NIH Image to ImageJ: 25 years of image analysis. Nat Methods. 2012;9(7):671-5.

13. Vlodavsky I, Miao HQ, Medalion B, Danagher P, Ron D. Involvement of heparan sulfate and related molecules in sequestration and growth promoting activity of fibroblast growth factor. Cancer Metastasis Rev. 1996;15(2):177-86.

14. Billings PC, Pacifici M. Interactions of signaling proteins, growth factors and other proteins with heparan sulfate: mechanisms and mysteries. Connect Tissue Res. 2015;56(4):272-80.

15. Hirabayashi S, Shigematsu H, lai M, Takashima S. A neurodegenerative disorder with early myoclonic encephalopathy, retinal pigmentary degeneration and nephronophthisis. Brain Dev. 2000;22(1):24-30.

\section{Publisher's Note}

Springer Nature remains neutral with regard to jurisdictional claims in published maps and institutional affiliations. 